\title{
6. Der revolutionäre Blick der Partisanenfotografie
}

\begin{abstract}
Neben den bereits erwähnten unmöglichen Bedingungen, unter denen sich die Partisanenfotografie entwickelte, hing ihre Produktion anfangs von den Druckereibesitzern ab, da es in dieser Zeit keine Maschinen für Drucksatz, Stereotypie, Guss und Rotation gab und man Zeitungen überwiegend mit Hilfe von Zyklostil-Maschinen druckte. ${ }^{1}$ Als Rotationspapier das Packpapier ablöste - das sich eher zum Verpacken von Geschenken als zum Drucken eignete, da die Farbe darauf häufig zerlief - war es kaum möglich, Zeitungen auf mehr als zwei Seiten zu drucken. Und gerade aus diesem Grund wurde eine große Zahl der Taschenzeitungen auf normalem Briefpapier mit der Hand verfasst, doch häufig fehlte auch hierzu das notwendige Material, nämlich Tinte und Füllfederhalter. Zudem gab es nur wenige Schreib- und Vervielfältigungsmaschinen. ${ }^{2}$ Die Kommunistische Partei Jugoslawiens stützte sich auf ihre Erfahrungen aus der Vorkriegszeit und die eigene technische Arbeitsweise während ihrer illegalen Tätigkeit.
\end{abstract}

Die Produktionsbedingungen der Partisanen und die Möglichkeit der fotografischen Dokumentierung waren auch beeinträchtigt, weil die Aktionen gegen den stärkeren und besser ausgestatteten Feind im Schutz der Dunkelheit ausgeführt wurden. Von einer solchen Erfahrung zeugt der Bericht des Stabs der 18. Division, in dem die Aktion der Sprengung der Eisenbahnlinie zwischen den slowenischen Dörfern Preserje und Goričica beschrieben wurde, die ein namentlich ungenannter amerikanischer Fotoreporter nicht fotografieren konnte:

„Die Explosionen zählte auch der amerikanische Fotoreporter begeistert mit. Er konnte nicht mit Hilfe von Magnesium fotografieren, da er mit dieser Art der Beleuchtung den Standort der Minenleger aufgedeckt hätte, die auf den Eisenbahnschienen lagen. Wahrscheinlich hätte er damit auch sich selbst zu erkennen gegeben und das Feuer aus Maschinengewehren und Mörsern auf sich gezogen. Zu diesem Schluss war er selbst gekommen und versuchte erst gar nicht zu fotografieren. Die Explosionen konnte er aus technischen Gründen nicht filmen. $\ll^{3}$

\footnotetext{
1 Zyklostil oder Gestetner sind Druckmaschinen, die mit Hilfe von auf Schreibmaschinen getippten Matrizen aus Papier arbeiten. 2 Hrvačić, Fabijan. Listovi, brošure i leci - ubojito oružje protiv neprijatelja u Pokuplju i Žumberku. In: Zatezalo (Hrsg.). Djuro Četvrta godina narodnooslobodilačkog rata na području Karlovca, Korduna, Like, Pokuplja i Žumberka. Karlovac: Historijski arhiv u Karlovcu 1981, S. 134.

3 Zbornik dokumenata i podataka o narodnooslobodilačkom ratu jugoslovenskih naroda. Bd. V/4: Borbe u Hrvatskoj 1944. godine. Belgrad 1961.
} 


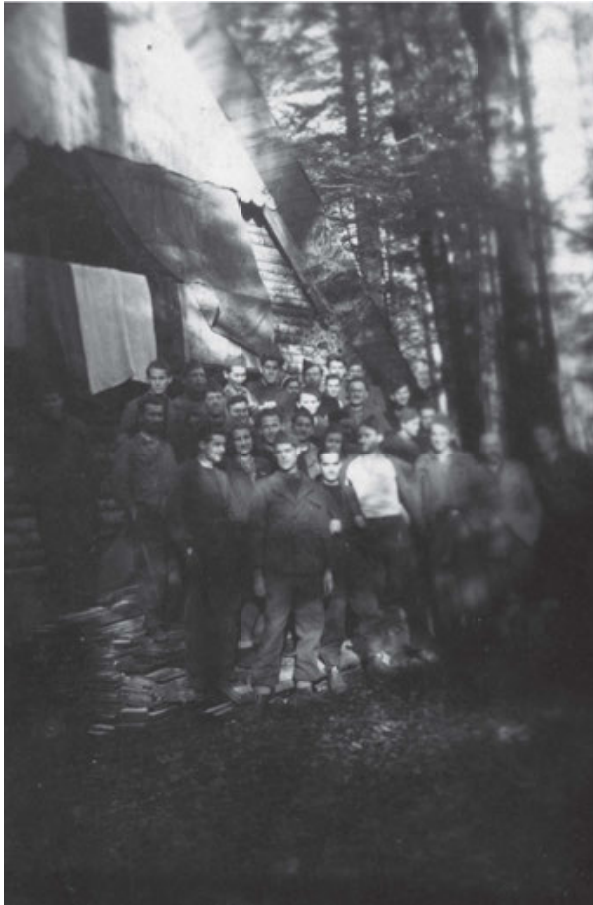

Das Kollektiv der Druckerei Naprijed (Vorwärts) in Drežnica, Kroatien, 1944. Foto: Marija Kreačić. Zagreb, Kroatisches Historisches Museum I HPM/MRNH-F-3517.

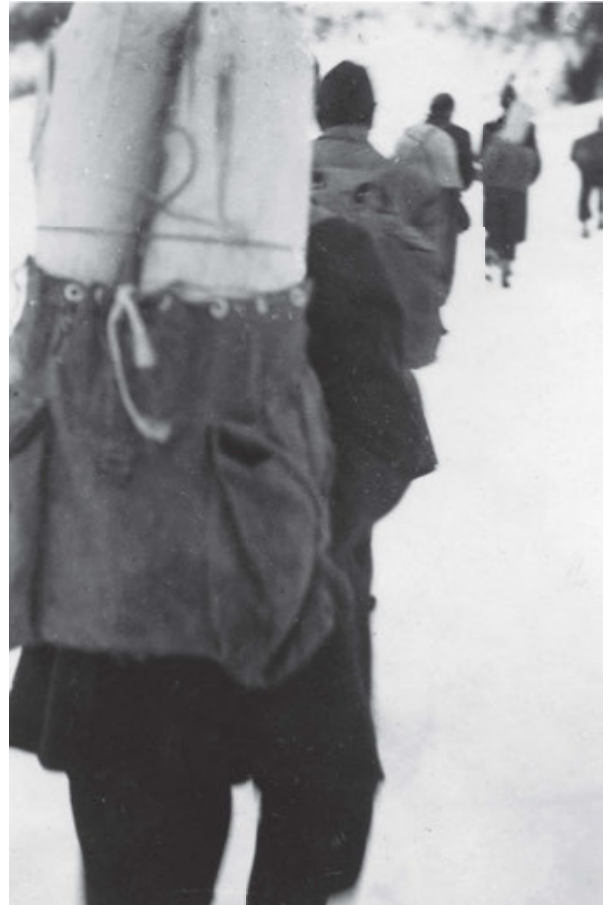

In der Kolonne. Fotograf unbekannt. Zagreb,

Kroatisches Historisches Museum | HPM/MRNHF-3569.

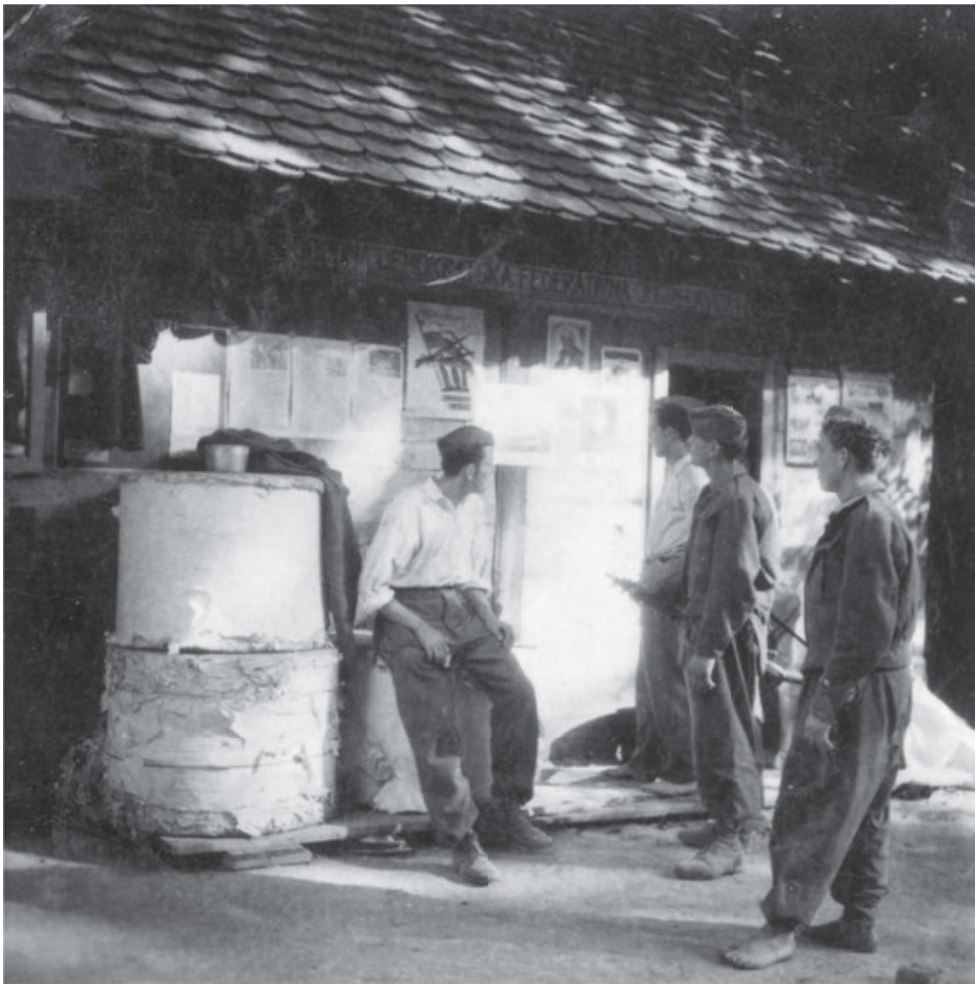

Sichergestellte Papierrollen nach einer geglückten Sabotageaktion auf einen Zug der Ustascha bei Koprivnica, Kroatien.

In der Nähe von Kordun, Kroatien. Fotograf unbekannt. Zagreb, Kroatisches Historisches Museum | HPM/MRNH-F-4808. 
Nachts wurden die Negative auch meist in zuvor mit Sand sorgfältig gereinigten Verpflegungsbehältern entwickelt und fixiert. Für die Filmentwicklung fehlte es an Behältnissen mit Deckel, Entwicklungsspiralen, Messbehältern, Thermometern und Filmen. Nicht einmal hohe Offiziere der Partisanenbewegung sind vom Mangel verschont geblieben, was aus dem Brief des obersten Kommandanten Josip Broz Tito an Svetozar Vukmanović-Tempo hervorgeht, in dem er ihn bat, neben Zigaretten auch Filme zu senden:

"Wenn die Filme, die ich nach Sarajevo geschickt habe, entwickelt sind, bitte ich dich, sie mir zu schicken, und wenn eine Aufnahme mehr oder weniger gut gelungen ist, so lass diese in möglichst großen Stückzahlen vervielfältigen. Besorge mir daneben auch Filme für meinen Fotoapparat; wenn es in Sarajevo noch Zigaretten gibt, und insbesondere der Marke 'Neretvar, vergiss die Armen hier nicht. $\ll^{4}$

Filme konnten nur in finsteren Nächten ohne Mondschein entwickelt werden; man trocknete sie mit einfachen Schwämmen und betrachtete sie im Schein des Feuers. ${ }^{5}$ All diese Bedingungen beeinflussten das finale Aussehen der Fotografien und wurden zu ihrem Referenzmerkmal, das die Partisanenfotografie auch der technischen Produktion der weitaus mächtigeren Propagandamaschinerie der deutschen Wehrmacht und der Ustascha entgegensetzte. Und gerade die Partisanenfotografie führte wegen der schwierigen Produktionsbedingungen zu einem Hacken des Produktionsprozesses. Man erdachte neue Formen der Produktion und - weitaus wichtiger - der Distribution, die nicht von oben nach unten, sondern umgekehrt, von unten nach oben, verlief. In erster Linie war dadurch eine schwächere propagandistische Kontrolle und eine Dezentralisierung der Propagandaorganisation in den ersten Jahren des Krieges möglich. Dank der Alphabetisierung und Schulung zur Verwendung verschiedener Technologien wurden die bis dahin stets sozial und politisch im Abseits stehenden Schichten gestärkt. An dieser Stelle gilt es zu erwähnen, dass die Fotoreportage, die nach dem Ersten Weltkrieg infolge der hoch professionalisierten Fotoreporterdienste entstand, zu einer Trennung des Objekts und Subjekts führte. Diese Regel wurde in Ausnahmezuständen jedoch von allen Seiten außer Kraft gesetzt, sodass eine »Objektivität« nicht zu verwirklichen war. Im Unterschied zu Fotoreportern, die auf allen Seiten der Militäreinheiten engagiert sind, legten die Partisanenfotografen oftmals ihre eigenen Regeln fest, während die Betrachter der Aufnahmen den Fotografen als ihr eigenes Surrogat ansahen, sich über Siege und Erfolge freuten und wegen Verlusten trauerten. ${ }^{6}$ Die Partisanenfotografie wurde auf eine andere Weise konsumiert, sie war nicht für das Bürgertum der westlichen Staaten bestimmt und auch nicht Teil der Werbung für bestimmte Produkte, sodass man gerade deswegen behaupten kann, dass die Partisanenfotografie den Kampf gegen Elend zum Gegenstand der Konsumierung gemacht hat. ${ }^{7}$

\footnotetext{
4 Zbornik dokumenata i podataka o narodno-oslobodilačkom ratu jugoslovenskih naroda. Bd. II/6: Dokumenta vrhovnog štaba Narodnooslobodilačke vojske Jugoslavije 1941-1942. Belgrad 1954.

5 Skrigin, Žorž. Rat i pozornica. Belgrad: Turistička štampa 1968, S. 23.

6 Rosler, Martha. Decoys and Disruptions: Selected Writings, 1975-2001. Cambridge und London: The MIT Press 2004, S. 226227.

7 Benjamin, Walter. Eseji. Belgrad: Nolit 1974, S. 107.
} 
Die fehlende Indexierung und Signierung der Fotografien kann man als eine Form der kollektiven Praxis ansehen. Für die meisten Fotografen war die Signatur unwichtig, weil sie die Arbeit als etwas Kollektives auffassten. Vor diesem Hintergrund ist insbesondere eine Fotografie interessant, die sich heute im Historischen Museum in Zagreb befindet und die auf den Negativen im Fotoapparat eines gefallenen Partisanen entdeckt wurde. Sie entstand während der Überquerung der zerstörten Brücke über den Fluss Neretva in der Bewegung, sodass sie zwar unscharf, jedoch gerade wegen der fehlenden Ästhetik besonders eindrucksvoll ist. Ihre mangelnde Qualität sagt auch etwas über die gesellschaftliche und wirtschaftliche Stellung ihres Schöpfers, und zwar auf die gleiche Art und Weise, wie dies heutzutage die ästhetisch minderwertige digitale Fotografie tut. Hito Steyerl zufolge würde man bei dieser den Fokus als Frage der Klasse und privilegierten Position werten, während er die Bildauflösung indes als eine unnötige Fetischisierung betrachtet. ${ }^{8}$

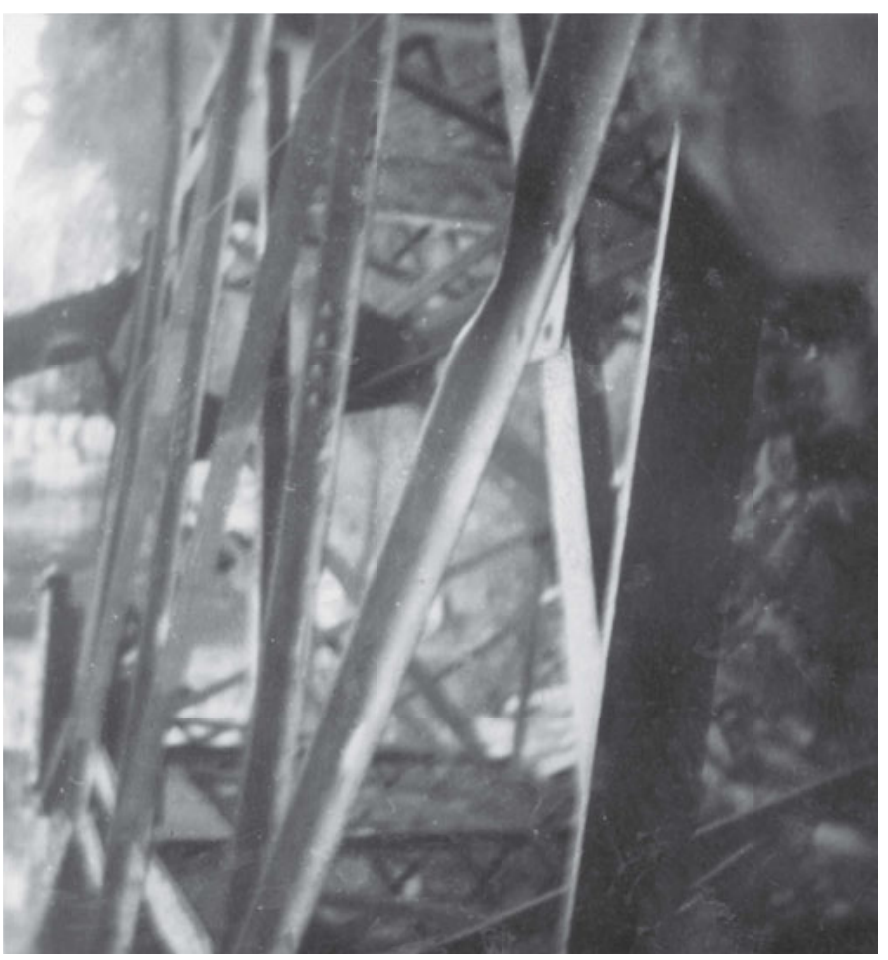

Übergang über den Fluss Neretva. Fotograf unbekannt. Zagreb, Kroatisches Historisches Museum I HPM-MRNH-F-830.

Der mittlerweile 95-jährige Franjo Cvrtila, der heute in Zagreb wohnt, ist einer der wenigen noch lebenden Partisanenfotografen. Er erzählt beispielsweise, von seinen Vorgesetzten niemals Befehle bekommen zu haben, was er fotografieren sollte. Einzig war ihm verboten, Opfer unter den Partisanen zu fotografieren, weil solche Bilder die feindliche Propaganda für sich nutzen konnte. Er schloss sich im Alter von nur 16 Jahren den Partisanen an und 
kämpfte in der 16. Jugendstoßbrigade Joža Vlahović. Nach dem Krieg wurde er Fotograf beim Dritten Kongress des Verbands der Antifaschistischen Jugend Jugoslawiens.

»Ich gehörte der Arbeiterklasse an. Als Diener hatte ich ein schweres Leben, man demütigte mich als Kind. Ich arbeitete als Lehrling in einem Malerbetrieb, doch dann begannen sie in Koprivica, Menschen zu erhängen. Meine Schwester arbeitete zu dieser Zeit für das Rote Kreuz, während meine beiden Brüder bei den Ustascha waren. Zu den Partisanen flüchtete ich 1943 mit meiner Mundharmonika und meinem Fotoapparat der Marke Kodak Retina. Bei den Kämpfen in Žumberak gegen die Verbände der Ustascha starben sehr viele von uns, und ich verlor all meine Fotografien. Vor den Kämpfen habe ich sie einer Krankenschwester gegeben, um sie für mich aufzubewahren oder zu verbrennen, wenn die `Bobaner k kommen sollten. Ich überlebte die Kämpfe, doch sie verlor ihr Leben, vernichtete aber vorher alle Fotografien. Es war verboten, tote Partisanen zu fotografieren. « ${ }^{9}$

In einer ähnlichen Situation befand sich auch Žorž Skrigin während des Krieges, als er sich im Jadovnik-Gebirge mit den Partisanen aus dem Siebten Korps, der sowjetischen und der britisch-sowjetischen Militärmission - zu der Randolph Churchill und Stojan Pribićević gehörten - von feindlichen Kräften umzingelt sah. Wegen der großen Gefahr, in Gefangenschaft zu geraten, erging der Befehl, alle persönlichen Dokumente und Fotografien zu vernichten.

»Ich war verblüfft. Die Negative vernichten? ... Dann verstand ich den Befehl und es schien mir logisch zu sein, dass der Feind nicht in den Besitz einer solchen Dokumentation gelangen durfte. Doch wie sollte ich Sachen vernichten, die ich so lange und mit großer Mühe aufbewahrt habe? ... Habe ich es denn nicht geschafft, sie zu retten und über den Fluss Bosna hinüberzuschaffen? ... Hatte ich denn nicht Glück, als ich sie erneut aus Drvar gerettet habe?... Und wie soll ich sie nun vernichten? ... Nein! Ich werde es nicht tun! Ich beschloss, bis zum letzten Augenblick zu warten. In meinem Kopf hatte ich schon einen Plan ausgeheckt: die Negative werde ich vernichten, indem ich sie mit meinem Maschinengewehr durchlöchern werde. $\ll^{10}$

Darüber hinaus konnte das fotografische Material in den Kämpfen leicht zerstört werden, und die Feuchtigkeit stellte ein zusätzliches Problem dar, da man feuchte Negative nicht sofort trocknen konnte und sie dann unwiderruflich verloren gingen. Während die Soldaten bei der Überquerung von Flüssen in den über den Köpfen getragenen Bündeln ihre Kleidung trugen, um sie trocken zu halten, waren die Fotografen oftmals gezwungen, angezogen durch das Wasser zu waten und die Ausrüstung, die Negative und die bereits gefertigten Abzüge hoch über den eigenen Köpfen zu tragen.. ${ }^{11}$ Aus diesen Gründen lagerte man das Fotomaterial oftmals an verborgenen Orten oder vergrub es in den Wäldern, wie der Bericht der Fünften operativen Zone an den Hauptstab der Widerstandsbewegung der Einheiten in Kroatien vom 29. Mai 1942 angibt. ${ }^{12}$ Beim Einreichen der Filme zur Entwicklung sollte auf

\footnotetext{
9 Aus dem Gespräch des Autors mit Franjo Cvrtila am 13.12.2017 in Zagreb.

10 Skrigin, Žorž. Rat i pozornica. Belgrad: Turistička štampa 1968, S. 257.

11 Ebd., S. 213.

12 Zbornik dokumenata i podataka o narodno-oslobodilačkom ratu jugoslovenskih naroda. Bd. V/4: Borbe u Hrvatskoj 1942. Belgrad 1954.
} 
dem Briefumschlag stets Folgendes notiert werden: »Film, nicht bei Tageslicht öffnen«. Filme und Fotografien wurden mit der Partisanenpost geschickt. Untergrundkämpfer hielten über geheime Kanäle während der gesamten Dauer des Krieges diesen Austausch aufrecht; neben Fotografien wurden zu Fuß auch Briefe, Zeitungen, Pakete und zahnärztliches Material transportiert..$^{13}$ Es war außerordentlich wichtig, dass die Negative nicht in feindliche Hände gelangten, weil dies die Familien der Partisanen, die auf dem besetzten Territorium zurückgeblieben waren, in tödliche Gefahr hätte bringen können.

Zahlreiche Negative wurden bei feindlichen Bombardierungen und Angriffen vernichtet. Im Falle der deutschen Bombardierung des Krankenhauses in Korenica wurden sogar 1.500 Negative zerstört, wie Franjo Mosinger in seinem fotografischen Tagebuch festgehalten hat. Die bedeutendste Fotosammlung der Partisanen ging beim Bombenangriff eines deutschen Fliegers am 27. November 1943 auf dem Glamoč-Feld verloren, als Ivo Lola Ribar getötet wurde und als auf dem Boden des brennenden Partisanenflugzeugs das gesamte Fotoarchiv, an dem Vili Šimunov-Barba und Ernst Grgić sowie mutmaßlich auch Žorž Skrigin monatelang gearbeitet hatten, den Flammen zum Opfer fiel. Die Fotografien sollten für die Illustrierung von Zeitschriften und die Veranstaltung von Ausstellungen bzw. Repräsentation der Partisanenbewegung vor den Alliierten zuerst in Kairo und später in London verwendet werden. Es verbrannten circa 300 Fotografien, ${ }^{14}$ die man vorher sorgfältig ausgesucht hatte. Moša Pijade betraute Skrigin schon am darauffolgenden Tag mit der Aufgabe, seine Fotografien erneut zu erstellen, die Vladimir Velebit und Miloje Milojević anschließend nach Kairo brachten. Zu dieser Zeit wurden einige seiner Fotografien in der britischen Tageszeitung News Chronicle und in der amerikanischen New York Times veröffentlicht. ${ }^{15}$

Im Laufe des Jahres 1944 intensivierte man die Programme für die Ausbildung von Propagandamitarbeitern und neuen Fotografen. Eines der besten Beispiele hierfür bildet vielleicht das Fotografski priručnik (Handbuch der Fotografie), das Leutnant Milan Štok, Leiter der Fotosektion der Propagandaabteilung des Siebten Korps, verfasst hatte und mit Hilfe der Zyklostil-Technik drucken ließ. Im einleitenden Kapitel wird erläutert, dass das Handbuch für Fotoreporter der Volksbefreiungsarmee bestimmt ist, die man mit dem Fotografieren wichtiger und interessanter Ereignisse betraute, während man die Laboruntersuchungen wiederum der Fotosektion des Hauptstabs überlassen hatte. Dieses wertvolle Dokument verdeutlicht gleich zu Beginn die Relevanz von Schulungen und Kenntnissen der Fototechnik.

"Die größten Erfolge werden sicherlich nicht ausschließlich mit Hilfe der Technik erzielt, doch für diejenigen, die mit ihr nicht vertraut sind, bleibt dieser Erfolg unerreichbar. In diesem Handbuch wird ein für die Fotoreporter dringend notwendiger Gegenstand behandelt (viele sind immer noch ziemlich ungeschickt beim Fotografieren), damit sie den Bedürfnissen der modernen Fotografie genügen können. [...] Ziel dieses Büchleins ist, dass sich jeder Fotoreporter mit seiner Kamera bestens vertraut macht und sie auch nachts zu gebrauchen imstande ist, dass er die Grundbegriffe und die wichtigsten Verfahren der Filmbeleuchtung genau kennt, dass ihm die Tie-

13 Konjhodžić, Mahmud. Različite veze. In: Tihić, Esad und Kalem, Momčilo (Hrsg.). Veze u NOB: Ratna sećanja 1941-1945. Bd. 4. Belgrad: Vojnoizdavački zavod 1981, S. 44.

14 Skrigin, Žorž. Rat i pozornica. Belgrad: Turistička štampa 1968, S. 216. 


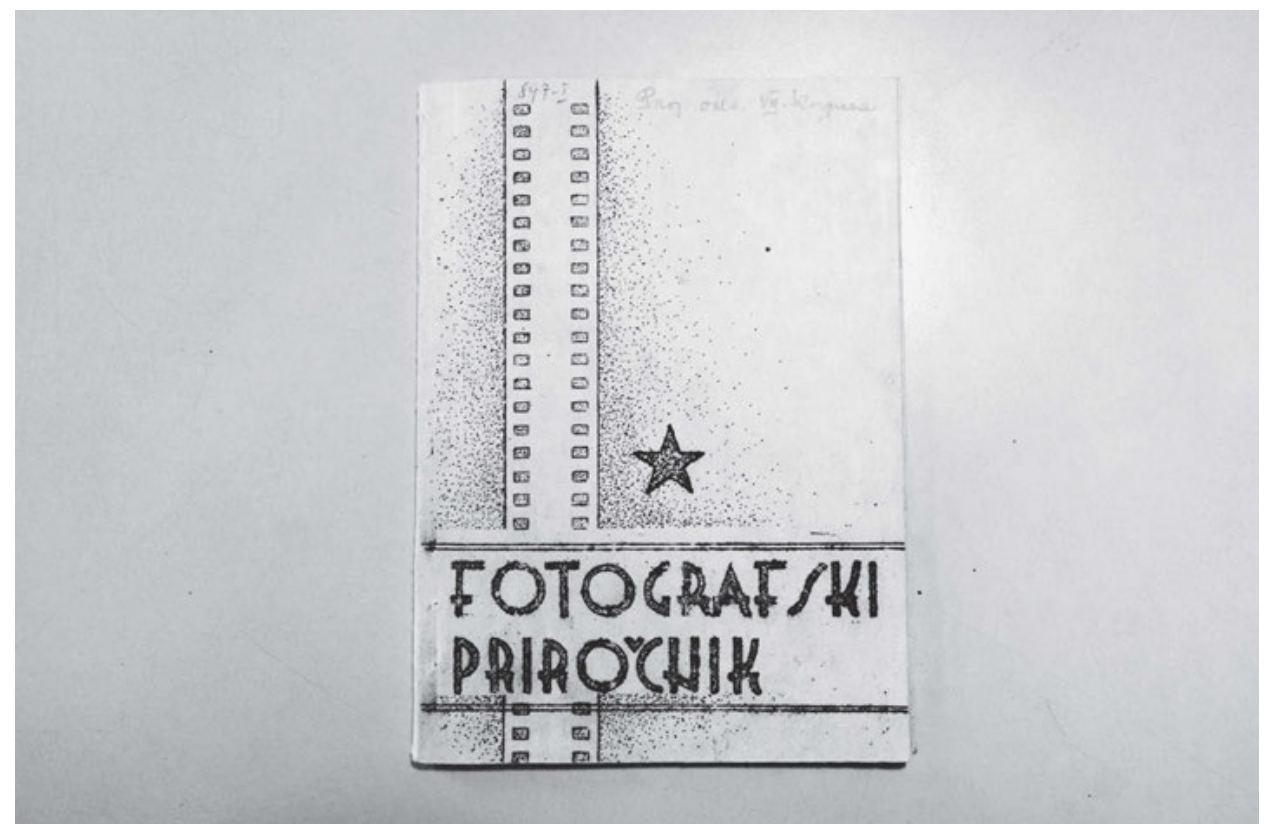

Titelseite von Milan Štoks Fotografski priručnik (Handbuch der Fotografie) für Partisanen. Foto: Davor Konjikušić.

fenschärfe bekannt ist, dass er sich mit dem Filmmaterial und seinen Charakteristiken auskennt und weiß, was ein Bild eigentlich ist. Dem militärischen Fotodienst kommt in unserer Armee eine besonders gewichtige Rolle zu. Er ist verantwortlich für die Verwahrung der Aufnahmen unseres gesamten Kampfes, sei es der kleinsten Aktionen, unserer Probleme und Leiden, aber auch der freudigen Momente in unseren Reihen, und insbesondere jetzt, da wir dem endgültigen Sieg so nahe sind. ${ }^{16}$

Dank dieses Handbuchs weiß man heute, dass in der Partisanenbewegung zumeist die Filmformate $24 \times 36 \mathrm{~mm}, 4 \times 4 \mathrm{~cm}, 6 \times 6 \mathrm{~cm}, 6 \times 3 \mathrm{~cm}$ und $9 \times 12 \mathrm{~cm}$ sowie die Kameras Leica, Contax, Kodak und Retina oder bei mittlerem Format Rolleiflex und Rolleicord verwendet wurden. In den Partisaneneinheiten, zumindest in den slowenischen Einheiten, fand man Apparate mit Filmspulen von $6 \times 9 \mathrm{~cm}$ sowie die US-amerikanische Kamera Kodak 35 Rangefinder, die die amerikanischen Streitkräfte 1938 für ihre eigenen Bedürfnisse weiterentwickelt hatten. In der Werbung nannte man die Kamera »zivile Miniatur «, sie war einst beliebt bei enthusiastischen Fotografen und wurde während des Krieges auch buchstäblich in die olivgrüne Soldatenuniform »eingekleidet«. Mit ihren technischen Eigenschaften und ihrem kompakten Design war die Kamera für die sich ständig in Bewegung befindlichen militärischen Einheiten höchst praktikabel. ${ }^{17}$ Štok betonte die Vorteile von Kameras mit einem 35-mm-Film, weil sie sogar 36 Aufnahmen ermöglichten und zudem eine größere Tiefenschärfe im Vergleich zu Fotoapparaten im Format $6 \times 9 \mathrm{~cm}$ aufwiesen, was sich vor allem für Aufnahmen während der Partisanenaktionen eignete. ${ }^{18}$

15 Ebd.

16 Štok, Milan. Fotografski priručnik. Propagandni odsjek Sedmog korpusa, o. 0. 1944, S. 3-5.

17 Life magazine, 08.05.1944, S. 59.

18 Štok, Milan. Fotografski priručnik. Propagandni odsjek Sedmog korpusa, o. 0. 1944, S. 7. 
Im Handbuch wird der Wahl des Bildausschnitts besondere Aufmerksamkeit geschenkt, wobei der Autor das oftmals zu weit entfernte Motiv, die ungeordnete Komposition und das immense "Durcheinander « auf den Partisanenfotografien als Problem ansah. Er schlug vor, jede Fotografie systematisch aufzubauen und sie mit einem zentralen Teil, der Umgebung und einem Hintergrund zu versehen. Mit Kameras kleineren Formats sollten vornehmlich die im Vordergrund stehenden Objekte und die Umgebung aufgenommen werden. ${ }^{19}$

»Im Winter sind kleine Objekte auf großen Schneeverwehungen aufzunehmen. Jede Aufnahme im Schnee muss einen ausgesprochen deutlichen Vordergrund aufweisen - Ski- oder Fußspuren, schneebedeckte Fichten oder Zäune usw. ... Die Sonne darf niemals ins Objektiv scheinen. Tipp: Aufnahmen so machen, dass mit der Hand oder mit Papier Schatten sichergestellt wird oder aus dem Schatten heraus fotografieren. $\ll^{20}$

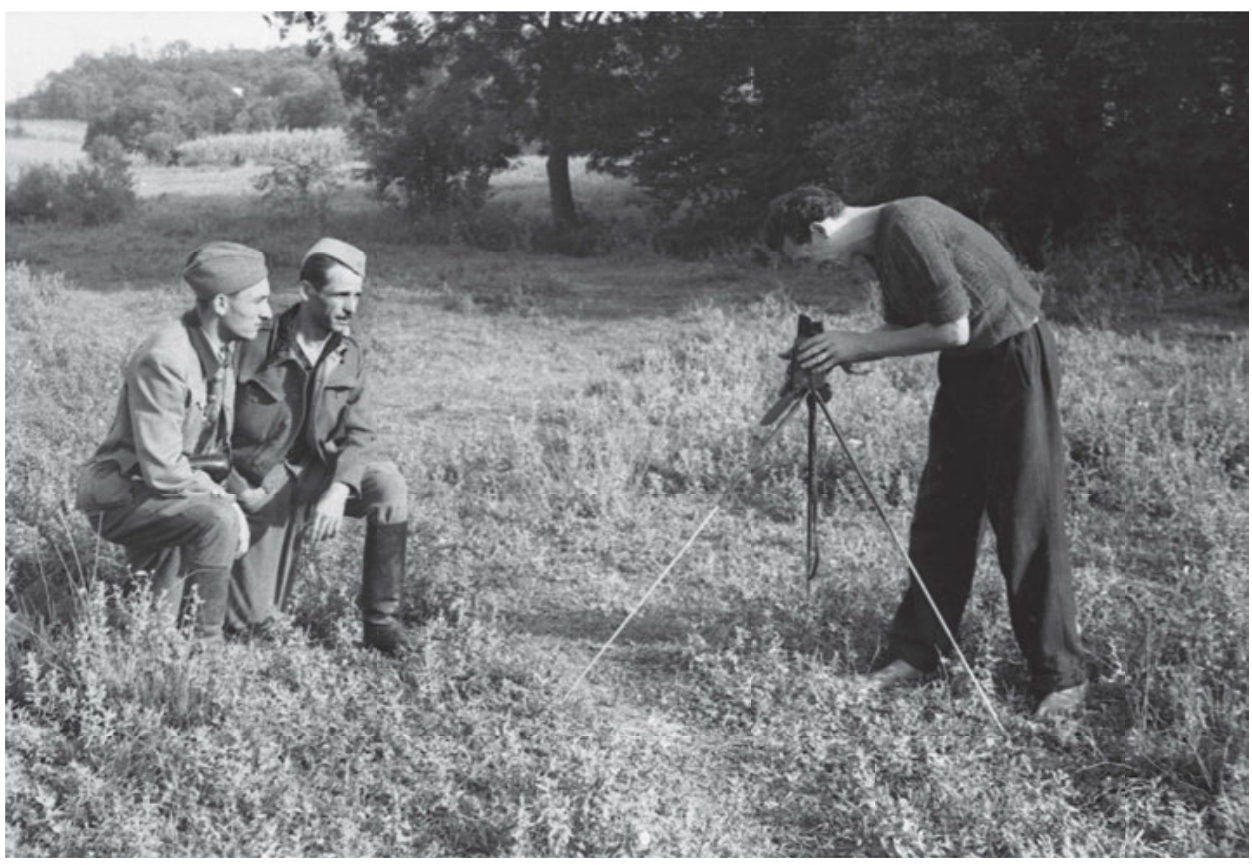

Fotograf bei den Partisanen in Breznica bei Slavonski Brod, Kroatien.

Zagreb, Kroatisches Historisches Museum | HPM-100522/36.

Der Text von Štok zeugt von einem Bildungsanspruch, ist präzise und leicht verständlich geschrieben. Jedem interessierten Leser vermittelt er auf einfache Art und Weise grundlegende technische Kenntnisse über Tiefenschärfe, Lichtmessung und Exposition, aber auch über komplexere physikalische Eigenschaften des Objektivs. Das vor über siebzig Jahren verfasste Handbuch ist hinsichtlich der analogen Fotografie noch immer aktuell. Štok verfügte offensichtlich über profunde Kenntnisse im Bereich der Physik; seine Erläuterungen zur Funktion der Blende, des Objektivdiaphragmas oder zur Entstehung der Fotografie sind auch heute zutreffend: 
»Mit der Belichtung der Filmschicht dringt Licht ein. Die bronzen-silberne Schicht war dem Einfluss des Lichtes ausgesetzt. Von außen wird auf der Filmschicht nichts verändert, das Bild ist immer noch unsichtbar und verborgen (latent). Erst durch die Entwicklung mit Hilfe von unterschiedlichen Entwicklern wird das Bild sichtbar, aber nur im Negativ: das, was in der Natur weiß ist, ist auf dem Negativ schwarz und umgekehrt. Negative werden auf fotosensitives Papier übertragen und dabei Bilder in Positive umgewandelt. Das Licht setzt den chemischen Prozess in der bromsilbernen Filmschicht in Gang, wobei dieser Prozess erst durch die Entwicklung in den Chemikalien zum Ausdruck kommt. Filmentwickler besitzen die Fähigkeit, Brom von den beleuchteten Bromsilberteilen zu unterscheiden und sich in Entwickler zu verwandeln, während sich das ursprüngliche Bromsilber in metallisches Silber verwandelt. Diese unzähligen Bromsilberteilchen bilden die Aufnahme. Die nicht beleuchteten Bromsilberteilchen bleiben vom Entwickler unberührt. Die Metallsilberteilchen sind die sogenannten Körnchen, und aus diesen Körnchen setzt sich dann das Bild zusammen. «21 $^{21}$

Das Handbuch belegt auch die häufigere Verwendung von panchromatischem Material im Unterschied zu orthochromatischen Filmen, was für die Partisanenfotografien von außerordentlicher Bedeutung war. Die panchromatischen Filme sind nämlich weitaus farbempfindlicher bzw. wellenlängenempfindlicher, was wiederum eine größere Bandbreite der Grautöne ermöglichte. Bei den orthochromatischen Filmen war es indes ausgesprochen schwierig, den roten Stern auf grünem Hintergrund zu isolieren und sichtbar zu machen. Im Gegensatz zu Farbfilmen, bei denen die Expressivität und der Kontrast auf Farben basieren, gründen Schwarzweißfilme ihre Expressivität auf dem Kontrast zwischen schwarzer und weißer Farbe bzw. auf der gesamten Tonskala. Štok widmete sich darüber hinaus eingehend der Frage, wie man bei der Entwicklung des Films feine Körner erhalten kann, und gab die optimalen chemischen Relationen zwischen unterschiedlichen Filmen und Entwicklern an.

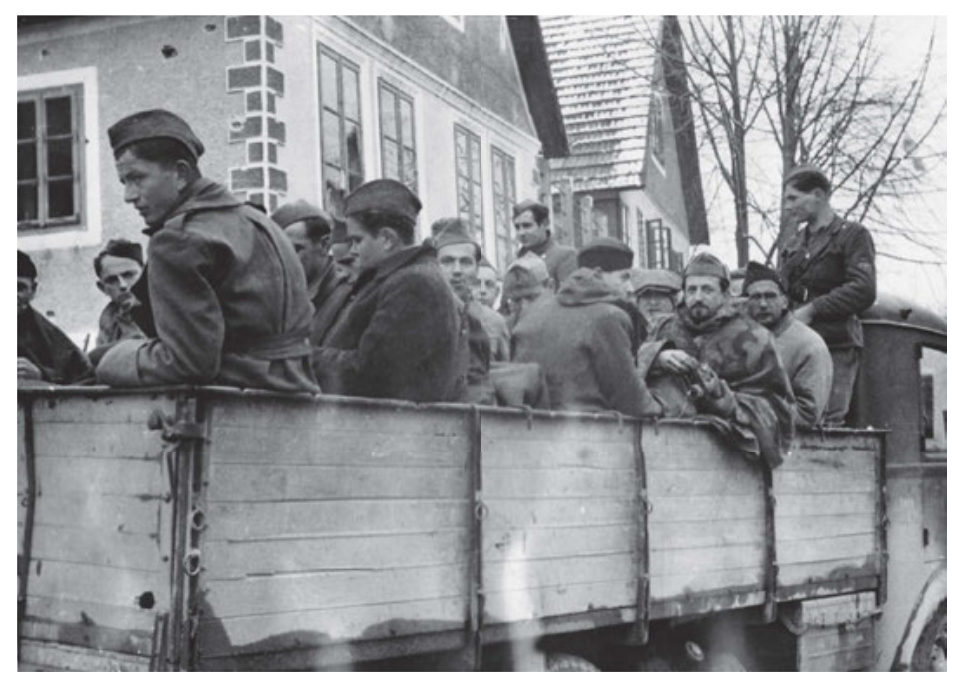

Zweite Sitzung des Antifaschistischen Rates der Volksbefreiung Jugoslawiens. Delegierte auf Lastern. Jajce, Bosnien und Herzegowina, 29.-30. November 1943. Fotograf unbekannt. Belgrad, Museum Jugoslawiens | III-180. 
Der wichtigste Teil des Handbuchs, das für die Untersuchung der Partisanenfotografie ein unentbehrliches Dokument darstellt, behandelt die Wahl des zu fotografierenden Motivs. Trotz der Ratschläge des Autors, wie man die Partisanenbewegung präsentieren sollte, erwartete er in der Wahl der Motive von jedem Fotografen Eigeninitiative und eigenen schöpferischen Anteil. Er riet, Kämpfe, das Antreten der Brigaden, Beratungen und Sitzungen sowie Kuriere, Waffen und Radiotelegrafisten bei der Arbeit aufzunehmen. Auf einer Seite stellte Štok die Propagandafunktion der Fotografie in den Vordergrund, um dann bereits auf der nächsten Seite über die künstlerischen Ausdrucksmittel zu sprechen: Sein Handbuch der Fotografie endet mit der Geschichte von Leonardo da Vinci. Die Betonung legte Štok auf das Fotografieren des Alltags der Partisanen, auf Augenblicke des Ausruhens und des Anscheins von Normalität.

Im Laufe des Jahres 1944 begannen die Partisanen im Rahmen der Hilfslieferungen der Alliierten auch größere Mengen an Fotomaterial zu erhalten, was eine raschere Entwicklung der fotografischen Tätigkeit ermöglichte. Der Wert der Warenlieferungen aus den USA, Großbritannien und Kanada belief sich bis zum 15. April 1945 auf 10.283.419 Dollar, wobei es sich vorwiegend um Lebensmittel, Schmieröle, Benzin, Petroleum, Ambulanzfahrzeuge, Konstruktionen für den Brückenbau, Material für Eisenbahnen, Kleidung und natürlich Fotomaterial handelte. ${ }^{22}$

Die Hilfslieferungen kamen aus Gorica, Triest, Mailand, Bari und Rom. Gleichzeitig konnten auch Feinmechanik-Werkstätten ihre Arbeit verstärken und nunmehr unterschiedliche Präzisionsinstrumente, einschließlich der Fotoapparate, reparieren. ${ }^{23}$ Deshalb ist auch nicht verwunderlich, dass die Hilfslieferungen der Alliierten mit der Entstehung des Fotografiehandbuchs zeitlich zusammenfielen. Trotz einer besseren Versorgung, die in erster Linie aus der politischen Anerkennung der Partisanenbewegung als einziger antifaschistischer Bewegung seitens der westlichen Alliierten resultierte, kam es dennoch sporadisch zu Engpässen bei der Belieferung mit Fotomaterialien. ${ }^{24}$ Sogar der bekannte slowenische Maler und Fotograf Božidar Jakac, Mitglied der 15. Brigade, blieb beim Fotografieren des Zweiten Treffens der Aktivisten der Befreiungsfront am 4. und 6. September 1944 in Črnomelj, dem mehr als 600 Delegierte beiwohnten, ohne Fotofilme..$^{25}$

Nicht nur die genannten materiell-technischen Bedingungen beeinflussten die Entwicklung der Partisanenfotografie. Das Leben der Partisanenfotografen, überhaupt das Leben aller Partisanen, war stetig bedroht. Zahlreiche Fotografen starben. Von vielen werden wir es nie erfahren, sie sind unbekannt geblieben. Viele von ihnen sind beim Fotografieren an vorderster Front ums Leben gekommen. Beim Versuch, den sich zurückziehenden Feind bei Lovas zu fotografieren, wurde der Fotograf Dragić Radivojević aus der Vierten Serbischen Stoßbrigade getötet. Ljubo Andjelić verstarb am 10. Juni 1943, als er das Bombardement der deutschen Luftwaffe in der Nähe des Ljuba-Grabes auf dem Berg Tjentište zu foto-

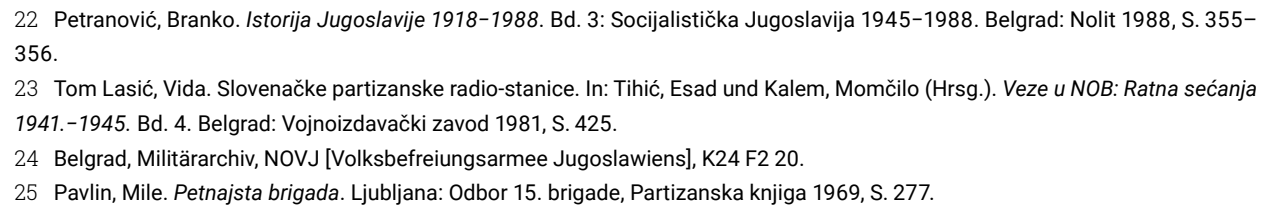




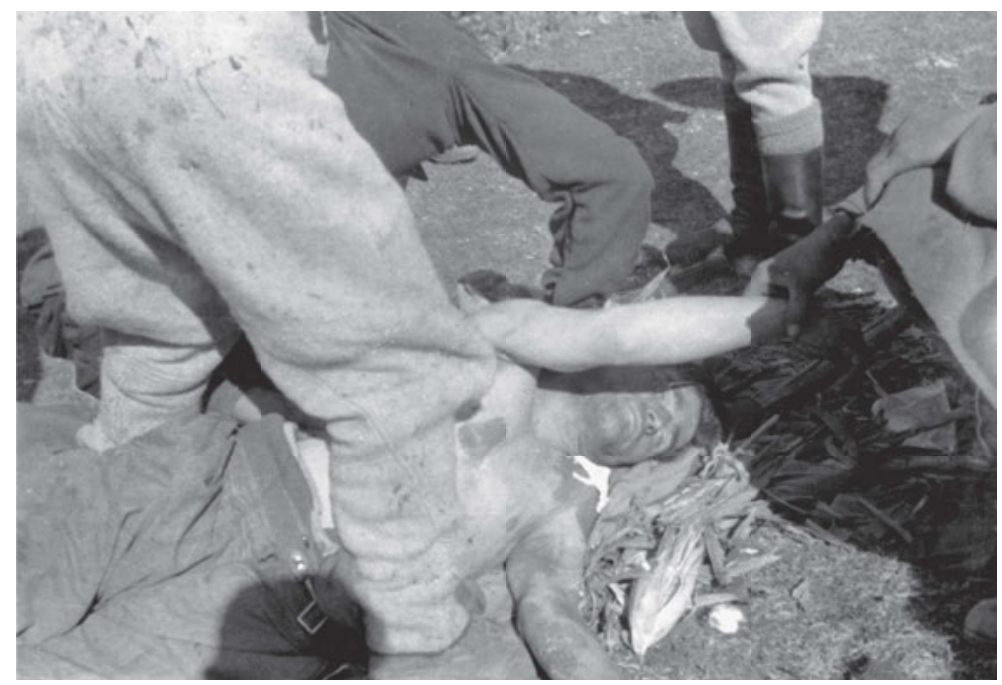

Schwerverwundeter Partisan der Ersten Dalmatinischen Brigade. Tal des Idbar, Bosnien und Herzegowina, 1943. Foto: Mladen Iveković. Zagreb, Kroatisches Historisches Museum I HPM-MRNH-A-6337/66.

grafieren versuchte. Dabei wurden auch die meisten seiner Fotografien vernichtet. Doch schlimmer als der Tod war manchmal das Leben in Zeiten des Hungers und der Krankheit, wovon folgende Schilderung bildhaft zeugt:

»... Als Erste begannen die Pferde tot umzufallen, weil es für sie fast gar keine Nahrung gab. Ihnen folgten die gefangenen Italiener und dann auch unsere Soldaten. Ich sah, wie Pferde starben und schon ein paar Minuten später in den Taschen der Gefangenen verschwanden. Von ihnen ließen sich auch unsere Soldaten dazu verleiten, obwohl wir uns niemals vorstellen konnten, dass unsere Leute verendetes Vieh essen könnten; auch das haben wir bei diesem Marsch erlebt. All diese Erlebnisse, Leiden und Verzweiflung wirkten sich auf die Entmutigten unter uns verheerend aus. Es war nicht selten der Fall, dass sich manch einer in seiner Verzweiflung selbst das Leben nahm. Ich sah mit meinen eigenen Augen, wie sich in der Kolonne, die von Krstač aus in das Idbar-Tal [unweit von Konjic, Bosnien und Herzegowina - Anmerkung D. K.] marschierte, ein Soldat (dessen Namen ich nicht kenne) eine italienische Granate unter die Jacke steckte und aktivierte; im Tal begegnete ich einem Mann, der zuerst seine Kameradin und dann auch sich selbst tötete; zwischen dem Gletschersee Boračko jezero und Konjic stieß ich nach einem der vielen schweren Kämpfe auf unseren verwundeten Kameraden Uroš Dmitrović (verwundet wurde er bereits in Banija), der apathisch und entkräftet auf dem Boden lag und fest entschlossen war, keinen Schritt mehr zu tun. Ich redete auf ihn ein, sich doch zu besinnen und weiterzugehen, doch er machte mir klar, dass es mit ihm zu Ende ging und er nicht weiter konnte. $\star^{26}$

Ganz andere Probleme existierten in den Einheiten selbst. Unter den Soldaten und den Fotografen kam es manchmal unnötig zu Spannungen, die oftmals tragikomische Situationen wie jene im Mostar-Bataillon auslösten: 
»Salko Zebić nahm zum ersten Mal in seinem Leben einen Fotoapparat in die Hände und schaffte es, ihn zu öffnen. Da er ihn nicht wieder schließen konnte, begann man Späße mit unserem `Fotoreporter zu machen, der danach den Fotoapparat wutentbrannt in den Bach warf. « ${ }^{27}$

Bei den Kämpfen im Rama-Tal, unmittelbar vor dem Kampf um die Befreiung von Prozor, verlor dieses Bataillon Luka Knežić, einen Fotografen aus Mostar und Kommunisten seit der Vorkriegszeit. Bevor er an Bauchtyphus erkrankte, schickte er Enver Ćemalović drei Filmrollen zu. Doch der Kommandant stieß bei der Suche nach irgendwelchen Papieren auf die Filmrollen und öffnete sie. »Alle drei Filme sind unwiderruflich verloren und ich bin mir sicher, dass dies die wertvollsten Fotodokumente über unser Bataillon waren. Als ich sah, was er getan hatte, stritten wir und schlugen uns fast. Luka hat in seiner durch Typhus verursachten Agonie Selbstmord begangen. ${ }^{28}$

Aus den schwierigen Produktionsbedingungen der Partisanenfotografie resultierten ihre Spezifika, ihre besonderen technischen Eigenschaften. Während des gesamten Krieges, insbesondere jedoch in den ersten drei Jahren, stand die Partisanenfotografie regelrecht am Rande der Existenz, doch mit der Zeit lernten die Partisanenfotografen, sich den Gefahren anzupassen und ihnen zu begegnen. Die Partisanenfotografie überwand ebenso wie die übrigen künstlerischen Ausdrucksformen der Partisanen ihre eigene Unmöglichkeit, was Miklavž Komelj am Beispiel der Partisanenpoesie eindrücklich zu zeigen vermochte. ${ }^{29}$ Die Fotografie teilte das Schicksal der Partisanenbewegung und ihrer Künste, hatte aber noch ein weiteres tragisches Los: Sie stand zwischen Dokumentation, Kunst und Propaganda. Die wichtigste Aufgabe der Partisanenfotografie jedoch war, trotz limitierter Ausrüstung, beschränkter Kenntnisse und Personalkräfte den antifaschistischen und revolutionären Kampf zu repräsentieren. In diesem Kampf gegen das Unmögliche gelang es den Partisanenfotografen im Gegensatz zu den Fotografen der Alliierten und der Entente im Benjamin'schen Sinne, ihr politisches Potenzial freizusetzen, was das Bündnis wie den Kampf stärkte und den Feind schwächte. Die Partisanenfotografie wurde mit all ihren technischen Unzulänglichkeiten innerhalb der Guerillabewegung und in einem asymmetrisch geführten Krieg zu einer Praxis der revolutionären Sichtweise.

27 Ćemalović, Enver. Mostarski bataljon. Mostar: Skupština opštine Mostar i Odbor za istoriju revolucionarnog radničkog pokreta i NOB-a Mostara 1986, S. 163.

28 Ebd., S. 175.

29 Komelj, Miklavž. Dva predavanja o partizanskoj umjetnosti. In: Jat: časopis studenata kroatistike, Bd. 1, Nr. 1, Juni 2013, S. 105. 


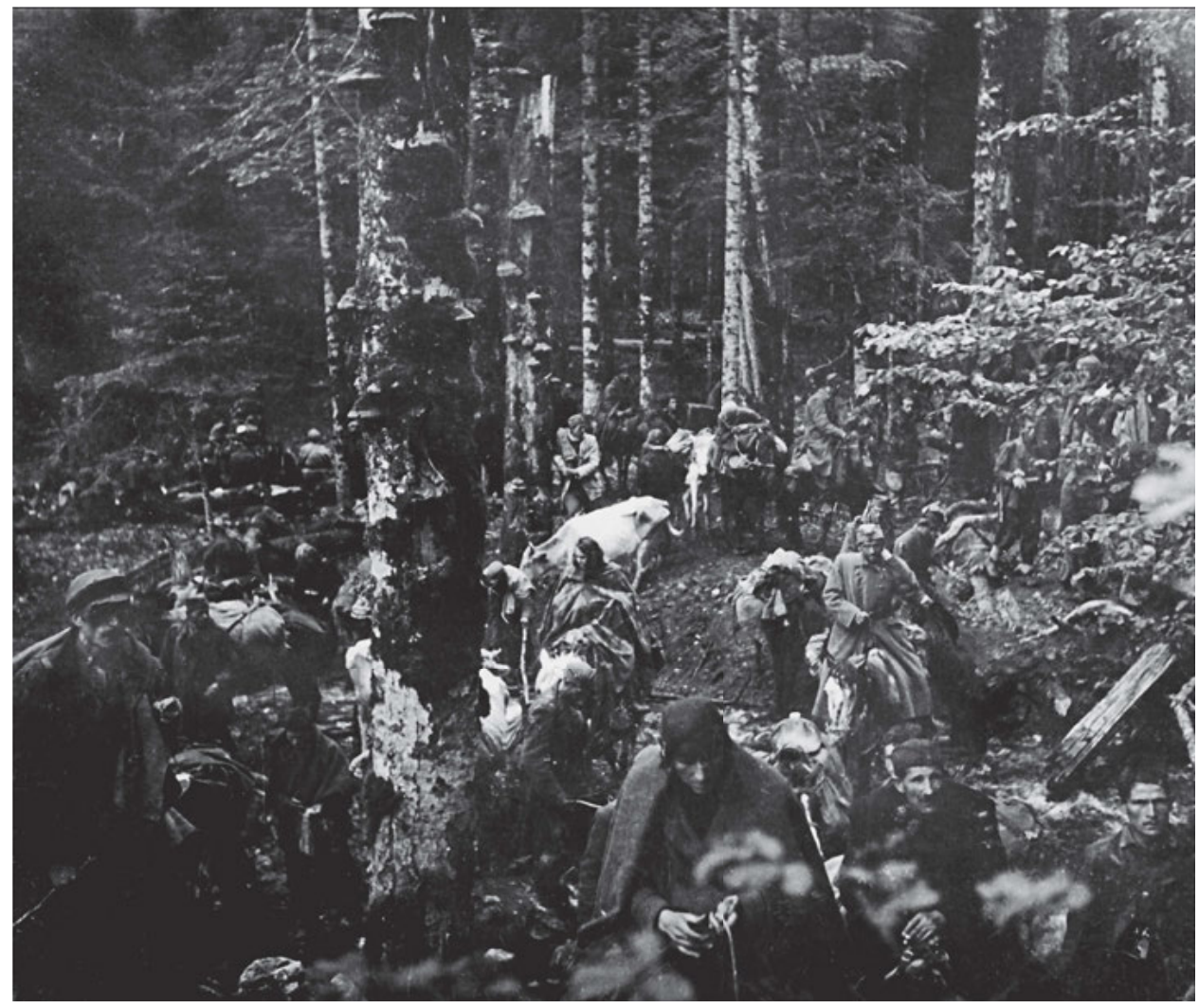

Kurze Rast der Verwundeten der Zweiten Division in der Nähe von Balinovac, Serbien. Fünfte feindliche Offensive (Schlacht an der Sutjeska), 1943. Foto: Žorž Skrigin. Belgrad, Museum Jugoslawien | III-3429.

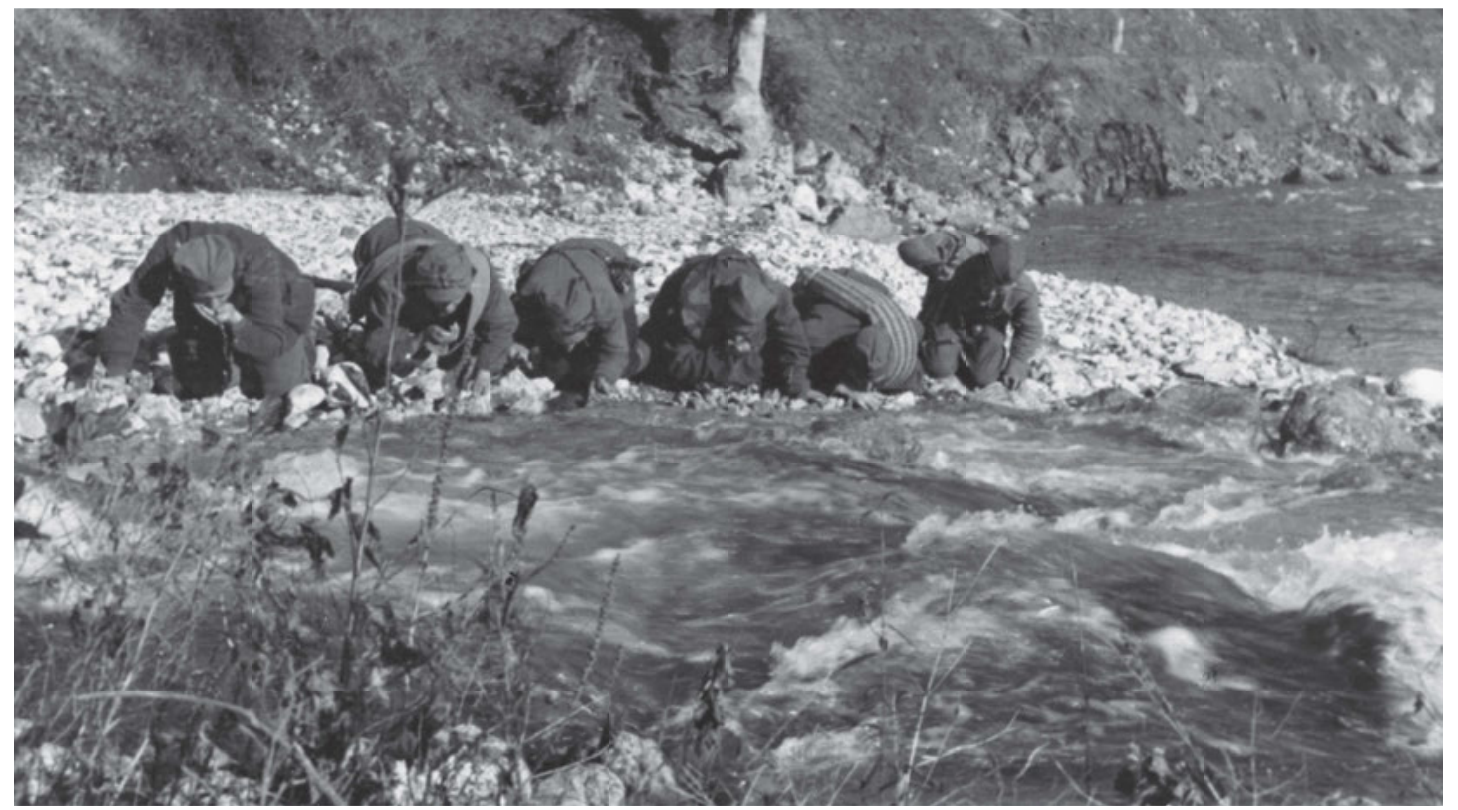

Partisanen trinken am Fluss. Kroatien, 1944. Fotograf unbekannt. Zagreb, Kroatisches Historisches Museum I HPM/MRNH-F-1056. 


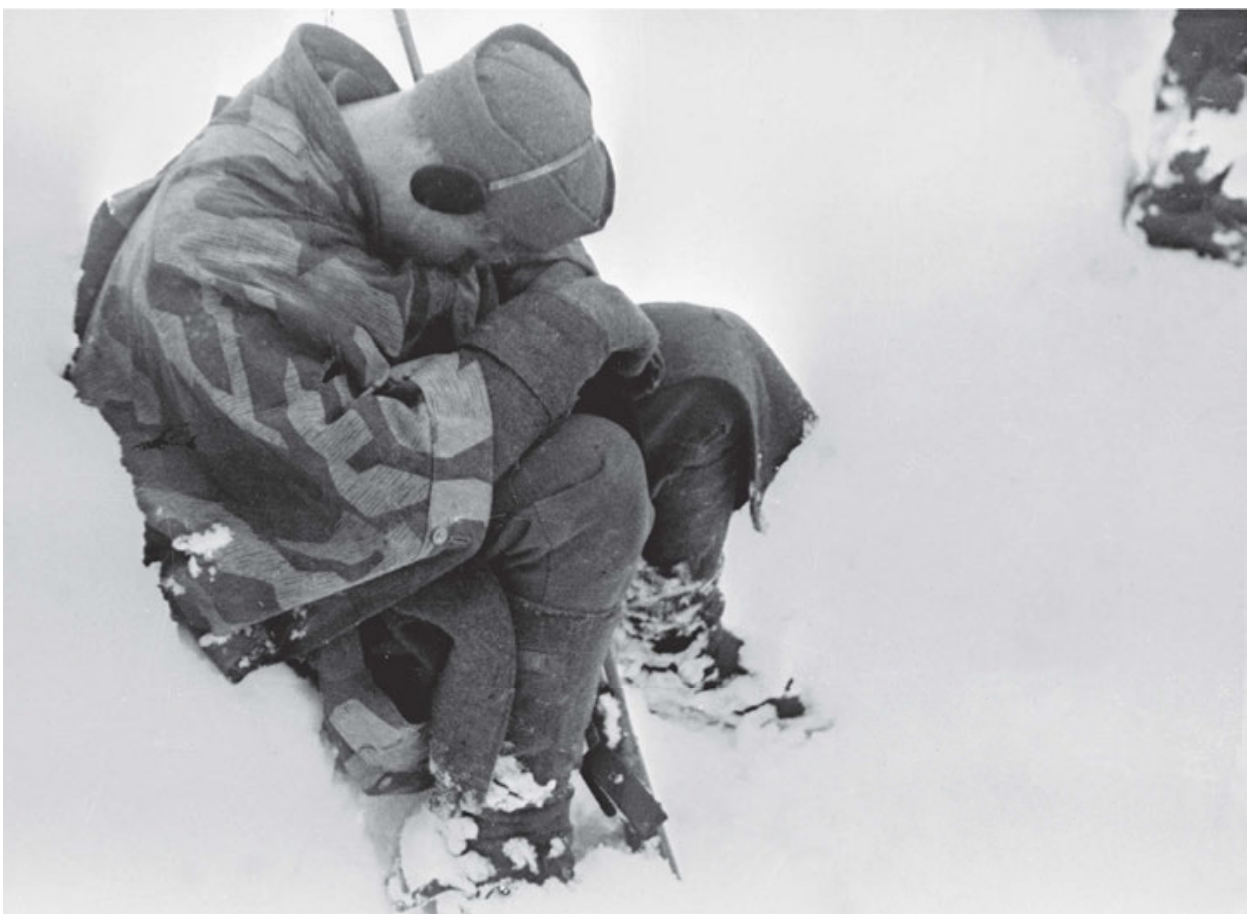

Angriff der 14. Division. Foto: Jože Petek. Ljubljana, Museum für Neuere Geschichte Sloweniens | PL1476.

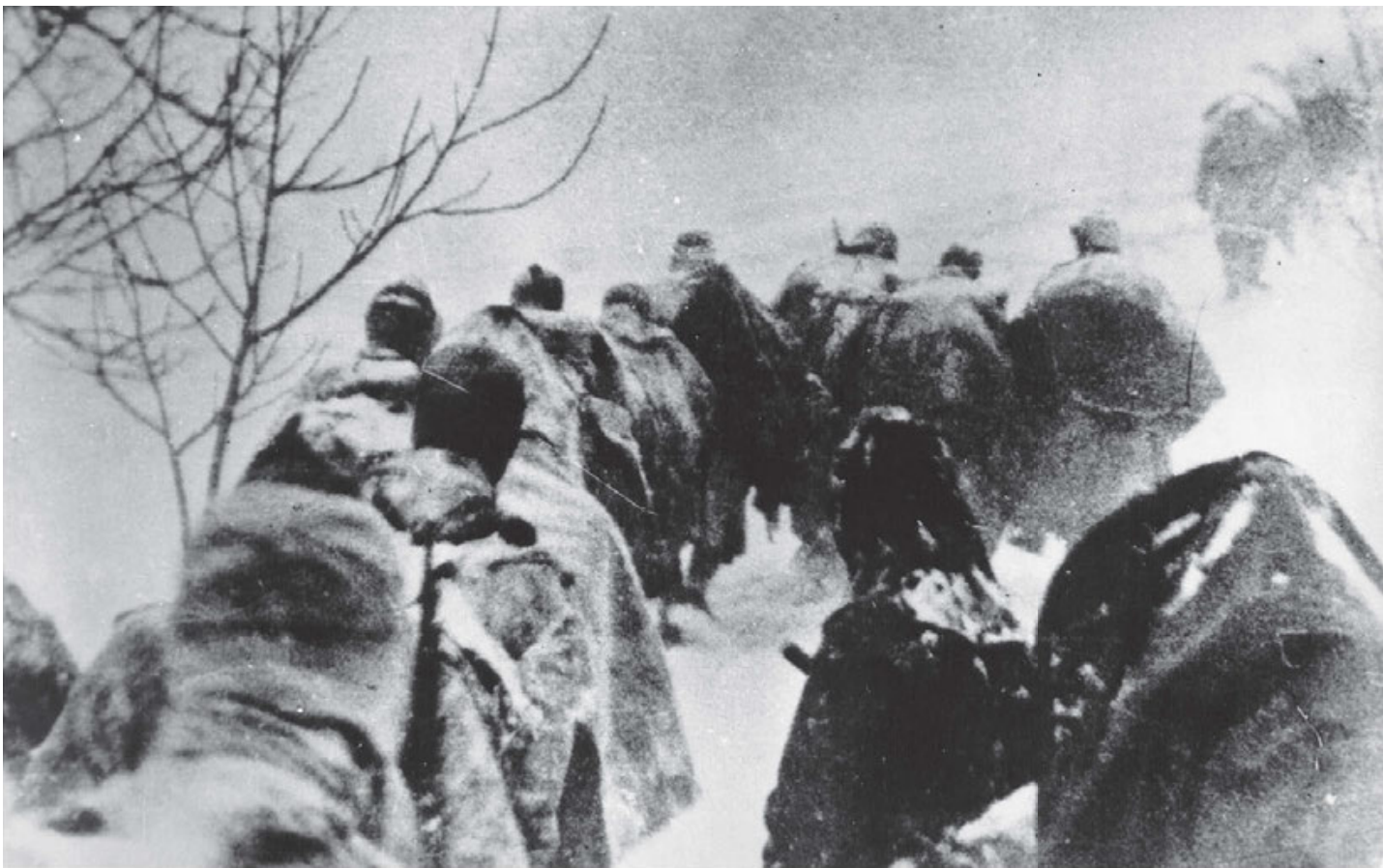

Evakuierung der Verwundeten, 1944. Foto: Jože Petek. Museum für Neuere Geschichte der Stadt Celje | FZ2 801-XIV. 